\title{
LETTERS
}

\section{SMART Designs in Observational Studies}

\author{
Jeffrey F. Scherrer, Ph. $D^{1,2,3}$, Sumitra Balasubramanian, $M S^{2,3}$, Kenneth E. Freedland, $P h D^{4}$, and \\ Patrick J. Lustman, Ph.D.4,5
}

'Department of Family and Community Medicine, Saint Louis University School of Medicine, St. Louis, MO, USA; ${ }^{2}$ St. Louis VA Medical Center, Research Service, St. Louis, MO, USA; ${ }^{3}$ Department of Internal Medicine, Washington University School of Medicine, St. Louis, MO, USA;

${ }^{4}$ Department of Psychiatry, Washington University School of Medicine, St. Louis, MO, USA; ${ }^{5}$ The Bell Street Clinic, John Cochran Hospital, St. Louis VA Medical Center, St. Louis, MO, USA.

J Gen Intern Med 29(7):986

DOI: $10.1007 / \mathrm{s} 11606-014-2859-0$

(c) Society of General Internal Medicine 2014

To the Editor - We appreciate Jackson and Gagne's ${ }^{1}$ enthusiasm for our recent study demonstrating that chronic opioid analgesic use (OAU) is significantly associated with risk of depression. ${ }^{2}$ We agree that utilizing medical record databases to inform clinical practice is possible. Their insights highlighted methodological challenges in studying opioid use and incident depression.

We concur that additional research is needed to improve depression diagnostic algorithms, including validation studies in which gold standard diagnoses are derived from structured interviews. We also agree that caution is warranted when identifying depression in patient populations in which depressive symptoms are not routinely monitored. As recommended by Townsend and colleagues, ${ }^{3}$ our depression outcome was identified in a health care system (Veterans Health Administration) in which annual screening is standard of care. We employed a published algorithm ${ }^{4}$ with high positive predictive value $(84 \%)$ that required two or more outpatient diagnoses or one inpatient diagnosis for depression in the same 12-month period.

Jackson and Gagne ${ }^{1}$ raise the possibility that propensity scores (PS) may not reflect treatment decisions when predictors of opioid therapy in the PS model include those measured up to onset of depression. In an ongoing study of OAU, depression, and incident heart disease, we are using PS models with predictors of opioid therapy up to the time of opioid initiation. These PS models perform as well as those using predictors of opioid therapy up to the time of disease onset in balancing patient characteristics across opioid treatment status. Additional information about propensity score (PS) weight distributions from our published analyses ${ }^{2}$ is provided in the next paragraph.

Published online April 23, 2014
Across the three opioid groups (1-90 days, 91-180 days and $>180$ days), mean PS weights were $1.0(\mathrm{SD} \pm 0.04)$; $0.99(\mathrm{SD} \pm 0.36)$ and $0.99(\mathrm{SD} \pm 0.36)$, and median $\mathrm{PS}$ weights were $0.99,0.90$ and 0.83 . PS weights ranged from 0.92 to 1.43 for opioid group 1-90 days; from 0.39 to 3.36 for opioid group 91-180 days, and from 0.18-6.91 for opioid group $>180$ days. Following Cole and Hernan's 5 suggestions, a mean of 1.0 with overall range from 0.18 to 6.91 is consistent with assumptions of PS weighting without bias due to truncating.

We believe that growing sophistication in methods and measurement will expand the use of observational cohorts and advance evidence-based medicine. Indeed, Jackson and Gagne point to the fact that observational cohort designs using medical record data can be the preferred choice over randomized controlled trials (RCTs) when alternative methods are unethical or not feasible in terms of time and money.

Corresponding Author: Jeffrey F. Scherrer, Ph.D; Department of Family and Community Medicine, Saint Louis University School of Medicine, 1402 S. Grand, St. Louis, MO 63104, USA (email: scherrif@slu.edu).

\section{REFERENCES}

1. Jackson JW, Gagne JJ. SMART designs in observational studies of opioid therapy duration. J Gen Intern Med. 2014;29:429-31.

2. Scherrer JF, Svrakic DM, Freedland KE, Chrusciel T, Balasubramanian S, Bucholz KK, Lawler EV, Lustman PJ. Prescription opioid analgesics increase risk of depression. J Gen Intern Med. 2014;29:491-9.

3. Townsend L, Walkup JT, Crystal S, Olfson M. A systematic review of validated methods for identifying depression using administrative data. Pharmacoepidemiol Drug Saf. 2012;21:163-73.

4. Frayne SM, Miller DR, Sharkansky EJ, Jackson VW, Wang F, Halanych JH, Berlowitz DR, Kader B, Rosen CS, Keane TM. Using administrative data to identify mental illness: what approach is best? Am J Med Qual. 2010;25(1):42-50.

5. Cole SR, Hernan MA. Constructing inverse probability weights for marginal structural models. Am J Epidemiol. 2008;168:656-64. 